Instituto Internacional de Investigación y Desarrollo Tecnológico Educativo INDTEC, C.A.

DOI: https://doi.org/10.29394/Scientific.issn.2542-2987.2021.6.21.14.267-281

OAI-PMH: http://www.indteca.com/ojs/index.php/Revista Scientific/oai

Ensayo Original / Original Essay

\title{
Tecnologías disruptivas en la transformación digital de las organizaciones en la industria 4.0
}

Autor: Edgar Eduardo Alayón Rodríguez Universidad Nacional Experimental "Rafael María Baralt", UNERMB ed.alayon@gmail.com

Cabimas, Venezuela https://orcid.org/0000-0002-6894-314X

\section{Resumen}

La transformación digital ya no es solo un cambio importante para las empresas de tecnología o startups (emprendimientos), esta se ha convertido en una importante ventaja competitiva para el posicionamiento de una empresa en un determinado mercado. Este ensayo se fundamenta en las investigaciones de autores como Arias (2018); Camargo-Vega, CamargoOrtega y Joyanes-Aguilar (2015); Rouhiainen (2018); y Tascón (2013). El objetivo de este ensayo presenta dos vértices, por un lado, pretende describir que tecnologías claves existen hoy y como implementarlas exitosamente en las organizaciones para lograr dicha transformación; por otro lado, intentar transmitir un pensamiento de innovación para poder aprovechar al mínimo las nuevas tecnologías. Lo que conlleva que se vea la transformación digital no como una simple implementación de nuevas tecnologías sino como "educación digital", ya que una vez que somos capaces de entender cómo funcionan también seremos capaces de poder aplicarla. Por ello es imprescindible entenderlas y saber cómo aplicarlas para avanzar hacia las siguientes tecnologías en la industria 4.0.

\section{Palabras clave: industria; inteligencia artificial; tecnología; transformación. \\ Código de clasificación internacional: 3311.01 - Tecnología de la automatización.}

\begin{abstract}
Cómo citar este ensayo:
Alayón, E. (2021). Tecnologías disruptivas en la transformación digital de las organizaciones en la industria 4.0. Revista Scientific, 6(21), 267-281, e-ISSN: 2542-2987. Recuperado de: https://doi.org/10.29394/Scientific.issn.2542-2987.2021.6.21.14.267-281
\end{abstract}

Fecha de Recepción: 18-01-2021
Fecha de Aceptación:

07-06-2021
Fecha de Publicación: 05-08-2021 


\title{
Disruptive technologies in the digital transformation of organizations in industry 4.0
}

\begin{abstract}
Digital transformation is no longer just an important change for technology companies or startups (entrepreneurship), it has become an important competitive advantage for the positioning of a company in a certain market. This essay is based on the research of authors such as Arias (2018); Camargo-Vega, Camargo-Ortega and Joyanes-Aguilar (2015); Rouhiainen (2018); and Tascón (2013). The objective of this essay presents two vertices, on the one hand, it intends to describe what key technologies exist today and how to implement them successfully in organizations to achieve said transformation; on the other hand, trying to convey an innovative thought in order to take advantage of new technologies to a minimum. This means that digital transformation is seen not as a simple implementation of new technologies but as "digital education", since once we are able to understand how they work, we will also be able to apply it. Therefore, it is essential to understand them and know how to apply them to advance towards the following technologies in Industry 4.0.
\end{abstract}

Keywords: industry; artificial intelligence; technology; transformation. International classification code: 3311.01 - Automation technology.

\section{How to cite this essay:}

Alayón, E. (2021). Disruptive technologies in the digital transformation of organizations in industry 4.0. Revista Scientific, 6(21), 267-281, e-ISSN: 2542-2987. Recovered from: https://doi.org/10.29394/Scientific.issn.2542-2987.2021.6.21.14.267-281

Date Received: 18-01-2021
Date Acceptance: 07-06-2021
Date Publication: 05-08-2021 


\section{Introducción}

Al analizar los cambios en las tecnologías de los últimos 30 años podemos observar que estos cambios han traído consigo una mayor disposición para innovar, crear o concebir algo aún más útil que lo anterior, hace 30 años era innovador pensar en dispositivos conectados de forma inalámbrica, y vemos que para hoy es algo trivial. Los teóricos de esta era digital aseveran que las nuevas tecnologías de ahora son: blockchain, inteligencia artificial, robótica, machine learning, realidad aumentada, computación en la nube, big data, internet de las cosas, entre otras.

Con estas nuevas tecnologías disruptivas surgen los siguientes planteamientos: ¿Qué podremos hacer con todas estas nuevas tecnologías disruptivas?; ¿Cómo se convertirán en fuentes de generación de nuevos productos y servicios?; ¿Qué tendrán que hacer las organizaciones para estar a la vanguardia o adaptarse a las tendencias tecnológicas que le envuelven?. La receta mágica para dar respuestas a todas estas cuestiones no existe, será por medio de la experiencia y la práctica lo que ayudará a evaluar que se está haciendo bien, por lo que el tiempo es un recurso decisivo ante estas interrogantes. Por este motivo, habrá que disminuir los periodos de experimentación y de prueba, para así poder tomar decisiones agiles y a la vez asertivas.

No cabe dudas que las nuevas tecnologías están afectando a las organizaciones, y es que los directivos de cualquier parte del mundo y de cualquier industria se tienen que plantear como enfrentarse a la 4 revolución industrial "la digital". Por ejemplo, el blockchain es una de las actuales tendencias, y para muchos empresarios esta ha pasado a ser un modelo de negocio como una forma de optimizar procesos o como apuesta de inversión para transformar la empresa; se puede ver el uso de la tecnología de blockchain en las criptomonedas, la cual está poniendo en aprietos a la industria bancaria. 
Así mismo, la realidad aumentada es otro ejemplo de tecnología disruptiva emergente, la aplicación de esta tecnología se está desarrollando el campo de la educación, en tiendas virtuales, videojuegos, sector inmobiliario, en los deportes, entre otros campos.

Estos ejemplos de nuevas tecnologías disruptivas tienen un patrón aplicable a todas las otras tecnologías de cómo afectan a las organizaciones, la cual podemos dividir en 4 aspectos:

1. Creación de un modelo de negocio basado en esa nueva tecnología.

2. La utilización de la tecnología como innovación de un producto o un servicio que ya existe.

3. El uso de la tecnología para ganar en eficiencia y optimización de procesos.

4. La aplicación de una tecnología pensada para una industria y usarla para otras.

De los anteriores aspectos podemos deducir que las nuevas tecnologías traen a las empresas nuevas oportunidades, y estará en manos de los directivos de estas aprovecharlas para mantener o generar un modelo de negocio que sea sustentable en la industria 4.0.

El objetivo de este ensayo es dar a conocer como estas nuevas tecnologías están realizando cambios de manera disruptiva y acelerada en diversos campos industriales, repercutiendo estos cambios en una transformación de la sociedad hacia la adopción de las tecnologías digitales.

\section{Desarrollo}

\subsection{Industria 4.0}

Es la época en la que vivimos en la actualidad, esta industria se caracteriza por tecnologías como la inteligencia artificial, internet de las cosas, la edición del genoma humano, la realidad aumentada, la robótica y la 
impresión en 3D. Es necesario recordar a Jacquez-Hernández y López (2018): quienes proponen la siguiente definición:

Es la utilización de nuevas tecnologías: sistemas ciber-físicos, Internet de la cosas e Internet de los servicios, etc., en el diseño, manufactura y comercialización de los productos, utilizando nuevos modelos de negocios que consideren la nueva dinámica entre dichas tecnologías y los empleados, así como entre las empresas, los proveedores y clientes (pág. 64).

\subsection{Tecnologías disruptivas}

\subsubsection{Big Data}

Mencionando a Tascón (2013): el Big data, "hace referencia a la cantidad -a la enorme cantidad, para ser más exactos- de datos, el tamaño y el número no son las únicas variables "gigantes" que están implicadas" (pág. 47); esto quiere decir que, consiste en analizar e interpretar grandes cantidades de datos, ya sean estructurados o no estructurados. Así miso, el Big Data aprovecha los datos almacenados de manera remota que pueden ser usados por las empresas para el proceso de toma de decisiones. Según Camargo-Vega, Camargo-Ortega y Joyanes-Aguilar (2015):

Las empresas que saben sacar provecho del Big Data pueden mejorar su estrategia y así permanecer en el mercado posicionadas, pues hará uso de nuevos conocimientos, con el gran volumen de datos o información que maneja a diario, que inicialmente no se les dio la suficiente importancia, por no tener una herramienta tecnológica que permitiera procesarla (pág. 75).

Como los conceptos antes mencionados se puede valorar que el big data tiene un enorme potencial para las organizaciones, ya que los datos se han convertido en el oro del siglo XXI, estos se han convertido en un recurso con una capacidad enorme, ya que para mejorar y validar tenemos que medir. Con el big data se puede obtener y almacenar grandes cantidades de datos de muchas fuentes distintas con el objetivo claro de poder entender patrones, 
Instituto Internacional de Investigación y Desarrollo Tecnológico Educativo INDTEC, C.A.

DOI: https://doi.org/10.29394/Scientific.issn.2542-2987.2021.6.21.14.267-281

OAI-PMH: http://www.indteca.com/ojs/index.php/Revista Scientific/oai

Ensayo Original / Original Essay

detectar ineficiencias y tomar decisiones complejas.

Esto es algo que se ha hecho desde hace tiempo atrás, en papel, en hojas de cálculos, u otros, la diferencia recae en que con el big data se pueden gestionar volúmenes enormes de datos que superan la capacidad de las herramientas convencionales a nivel de usuario, así como la facilidad y velocidad para realizar esos análisis de datos.

En el mundo empresarial y la adaptación a las nuevas tecnologías, el big data significa poder monitorizar y analizar datos del consumidor, tendencias de compras, sentimientos, asegurar la lealtad de los clientes, entre otras; se calcula que seis millones de personas están trabajando hoy en big data con una inversión cercana a los 57 mil millones de dólares, debido a que el volumen de datos generados en los últimos 3 años es el equivalente a los de toda la historia de la humanidad.

\subsubsection{Realidad aumentada}

Al respecto, Maquilón, Mirete y Avilés (2017): describen la realidad aumentada como:

[...] La ampliación artificial de la percepción de la realidad, por medio de información virtual. Dicha información virtual es generada con técnicas asistidas por ordenador y representada mediante los componentes tecnológicos específicos. La RA puede abordar todos los sentidos humanos de la percepción, sin embargo la variación de RA más extendida comúnmente es la representación de información virtual visual añadida al entorno real" (pág. 185).

Es necesario aclarar la diferencia entre realidad virtual y realidad aumentada, la primera se refiere a mostrar información digital en el mundo real, y la otra puede aplicarse en un dispositivo en la propia persona como en las Google Glass o bien en un objeto, por otro lado, realidad aumentada significa trasladar al usuario en un mundo virtual, en general requiere de un 
dispositivo de reproducción. En los últimos años se ha visto como importantes organizaciones de talla mundial como Disney, Fox, Microsoft, Facebook, Apple, Nasa, entre otras, están invirtiendo en estas tecnologías, el objetivo es poder transformar sus servicios para usuarios cada vez más exigentes que desean contenidos muchos más dinámicos.

Esta tecnología aplicada en los negocios trae consigo tres tipos de necesidades: en primer lugar, el desarrollo de plataformas para crear proyectos de realidad aumentada o virtual, compuestos principalmente por software, en segundo lugar, compañías desarrollando aplicaciones para esta tecnología, y en tercer lugar, soluciones de llave en mano para aplicar esta tecnología a una empresa consistiendo en las dos anteriores.

Uno de los desafíos de esta nueva tecnología en la actualidad se encuentra en compensar el balance entre funcionalidad y precisión con inversión necesaria; por ejemplo, la realidad aumentada aplicada a la industria inmobiliaria debe encontrar el balance entre la calidad en la visualización y el coste que va a suponer para la empresa y por ende su reducción o no de su margen de ganancia.

\subsubsection{Blockchain}

Por su parte, Arias (2018a): precisa el blockchain o cadena de bloques:

[...] Como un protocolo diseñado para darle el tránsito y la trazabilidad a operaciones virtuales. [...] El blockchain como su mismo nombre lo indica es una serie de bloques que contiene cierta información, esta información no se puede alterar, cambiar o modificar después de aceptada en el bloque ya que cada uno de estos bloques se identifica de manera consecutiva de acuerdo al bloque anterior (págs. 10-11).

Se podría decir que el blockchain es una base de datos de activos digitales distribuida en millones de computadores, la cual no pude ser alterada ni corrompida. La funcionalidad de la cadena de bloques es tan amplia que se 
Instituto Internacional de Investigación y Desarrollo Tecnológico Educativo INDTEC, C.A.

DOI: https://doi.org/10.29394/Scientific.issn.2542-2987.2021.6.21.14.267-281

OAI-PMH: http://www.indteca.com/ojs/index.php/Revista Scientific/oai

Ensayo Original / Original Essay

ha reverenciado como una revolución en internet, donde se han descubierto ciertos beneficios para aplicarla en otros temas. Para Arias (2018b):

Su función inicialmente es ser como un libro de registros, donde quedan inscritas todas las transacciones hechas por esta cadena, sus principales rasgos son el anonimato de las personas que tienen actividad en la cadena de bloques y la veracidad de cada transacción sin ninguna entidad intermediadora, ya que los mismos usuarios de la red hacen de este proceso claro y seguro (pág. 11).

La idea más poderosa de esta tecnología es el hecho de que la base está distribuida y la confianza se deposita en la colaboración en masa y no en una institución. No hay duda de que el potencial uso del blockchain es inimaginable para poder retirar organizaciones de mercados monopolísticos $u$ oligopólicos y para dar pie a una economía más colaborativa, además en términos de seguridad es uno de los sistemas más robustos que existen, con lo que empresas por ejemplo implementando proyectos de big data o loT (internet de las cosas) podrán incorporan el blockchain como sistema de descentralización y asegurar la información compartida.

\subsubsection{La nube}

Actualmente existe un aumento de Software como servicio, Plataforma como servicio e Infraestructura como servicio en la nube, conocidos en el idioma inglés como Software as a Service (SaaS), Platform as a Service (PaaS), y por último, Infrastructure as a Service (laaS); estos tres tipos de servicios, a la vez son modelos de negocios lanzados por compañías como Google, Amazon, Microsoft y Cisco, entre otras, atraen a clientes de varios sectores para poder utilizar fácilmente los beneficios de la nube externalizando la infraestructura necesaria y el software requerido.

Una de las ventajas de la nube o del Cloud Computing es el elevado nivel de poder de procesamiento en comparación con el procesamiento local, 


\section{Ensayo Original / Original Essay}

debido a poder externalizar el servicio se reduce el coste de infraestructura y así no tener que invertir en hardware/software, así como el ahorro en energético que conlleva.

La escalabilidad es otra ventaja de esta tecnología ya que permite pagar solo por lo que se usa sin necesidad de realizar grandes inversiones, permitiendo que varios empleados puedan acceder a la información y colaborar entre ellos desde distintas localidades. Cada vez más herramientas de negocios como la Planificación de recursos empresariales (ERP) o la Gestión de relaciones con el cliente (CRM) están ubicadas en la nube, lo que aporta seguridad y eficiencia en costes y generar análisis más allá de los datos generados. Los directivos deben plantearse el uso de la nube como parte de su organización debido a la mayor eficiencia que esta ofrece, la seguridad, integración de procesos, y así evitar costes fijos y grandes inversiones.

\subsubsection{Robótica}

Se pueden encontrar diferentes aplicaciones de la robótica en la empresa, las más destacada es la mejora en la productividad, la eficiencia en la productividad en la industria o en procesos donde se repiten tareas tienen como principal necesidad la automatización de procesos. La automatización es uno de procesos más apreciados por las empresas debido al ahorro en costes y la reducción de errores en forma exponencial. Sin embargo, hay que considerar no solo la posibilidad técnica sino también la viabilidad económica. ¿Cuánto costaría automatizar una tarea que quizás su coste con actividad humana pueda ser inferior?.

Otra aplicación seria la realización de tareas de alto riesgo, es el trabajo perfecto para un robot, ya que si es necesario este podría tomar riesgos que un humano no asumiría. En el ámbito empresarial un robot podría convertirse perfectamente en un asistente administrativo, capaz de procesar gran cantidad de información, los usos potenciales no tienen fin en esta etapa en que la 


\section{Ensayo Original / Original Essay}

robótica en la empresa está en sus comienzos.

\subsubsection{Inteligencia artificial}

Para Rouhiainen (2018): "[...] la IA es la capacidad de las máquinas para usar algoritmos, aprender de los datos y utilizar lo aprendido en la toma de decisiones tal y como lo haría un ser humano" (pág. 17). La inteligencia artificial actualmente se está posicionando dentro de las empresas como la responsable de ayudar en el proceso de tomas de decisiones, de anticiparse y colaboraren la reinvención del modelo de negocio, e incluso de rediseñar la experiencia del consumidor.

Entre las industrias con más potencial de integrar la inteligencia artificial esta la logística, teniendo en cuenta que se debe analizar los millones de mercancías que se mueven diariamente, y tomar decisiones como envíos, retrasos, mantenimientos de flotas de vehículos, retrasos entre otros.

Seguidamente el sector sanitario podría transformarse radicalmente si se es capaz de obtener patrones y tendencias de plagas o enfermedades, y así poder anticipar las enfermedades y que se debe hacer para prevenirlas. Otro sector que se puede beneficiar de la inteligencia artificial es la ciberseguridad, al buscar tendencias de actividades en la red y evitando así ataques a los sistemas informáticos. La industria automotriz es otra industria que tiene un potencial de disrupción enorme al utilizar esta tecnología, así lo está demostrando la empresa Tesla, Inc. y Google, LLC. con sus nuevos vehículos autónomos.

\subsubsection{Bots}

Los Bots o también llamados Web-bots, son aplicaciones de software que realizan tareas automatizadas en el ámbito de internet, estos surgieron gracias a la inteligencia artificial principalmente como funciones de los motores de búsquedas web con el objetivo de traer al usuario del bot la información que 
está buscando con la mayor precisión posible. Existen bots buenos y malos, los buenos por ejemplo son los rastreadores web y los malos aquellos que buscan correos electrónicos por internet para luego enviar spam o campañas publicitarias.

En los últimos años y gracias a la inteligencia artificial los bots se están haciendo aún más inteligentes, por ejemplo, son capaces de dar conversación y responder a preguntas en una página web, capaces de escribir en redes sociales, entre otros. En lo que concierne al mundo de la empresa y con el auge de las plataformas de mensajería muchas empresas que prestan servicios a través de internet se plantean incorporan el chatbot, es decir, un robot capaz de sostener una conversación con los usuarios que visitan una página web.

Un ejemplo del uso de los bots se puede observar en la empresa de la red social Facebook, la cual aprovechando esta tecnología lanzo una aplicación tipo chatbot para su aplicación Messenger y rápidamente generó una solución para que las empresas pudieran hablar con sus clientes sin intervención de algún humano y además personalizado a cada tipo de usuario. Los bots son una solución para que las empresas puedan volverse más digitales y activas en internet, pero a la vez es un modelo de negocios para algunos visionarios.

\subsubsection{Machine learning}

Lo maravilloso del machine learning radica en que fue desarrollada originalmente para que los computadores pudiesen aprender sin ser programadas, con lo cual emularía al cerebro humano para que pueda tomar decisiones y así automatizar procesos y empresa.

Con estas características, esta tecnología tiende a tener un número ilimitado de aplicaciones para cualquier empresa, actualmente ya existen muchos casos de usos en diferentes sectores, por ejemplo, una computadora 
de IBM ganó un campeonato de ajedrez contra un ser humano, las agencias de seguridad e inteligencia utilizan datos para hallar a los criminales por medio de Machine learning. Disney analiza las emociones del público ante diferentes escenas para realizar películas que no hieran los sentimientos de los espectadores y tengan el mejor final feliz posible, incluso YouTube utiliza el Machine learning para detectar videos que infrinjan sus políticas y eliminarlos directamente.

$Y$ es que el Machine learning requiere de relativamente pocos esfuerzos para poder aprender a realizar análisis de hechos, imágenes, procesos u operaciones de forma muy profunda y a la vez técnicamente perfecta basada en patrones y datos reales. Esta tecnología va de la mano con el big data y el internet de las cosas ya que para aprender necesita datos recopilados por objetos inteligentes que terminaran creando grandes bases de datos, es decir, big data y que podrán ser analizados para ver tendencias y proporcionar recomendaciones de mucho valor añadido.

\section{Conclusiones}

Es una decisión estratégica reflexionar que es lo más prioritario para las organizaciones y que resultados se desean obtener, esto ayudará a decidir cuales tecnología cumplirán con las expectativas que se buscan.

Una vez identificado el problema se estudia que tecnología podría ayudar a solucionarlo, y una buena forma es comprobar si ya existe un caso de uso avanzado en la misma línea, tratar de responder interrogantes como: ¿Que no está funcionando?; ¿Qué se puede cambiar al utilizar determinada tecnología?; ¿Cómo se medirá el éxito?; ¿Cuáles son las prioridades: Funcionalidad, coste, accesibilidad servicios, etc.?; y así externalizar o desarrollar la solución focalizándose en los aspectos más relevantes de los proyectos.

Implementar un proyecto tecnológico no es una decisión sencilla, tanto 
por el impacto económico e inversión de recursos como por la dificultad e implementarlo con éxito por lo que son muchas las grandes corporaciones que mantienen un diferencial importante entre inversión en tecnología y la habilidad de ponerlo en marcha.

Para cerrar ese diferencial entre inversión y puesta en marcha puede reducirse con un buen análisis y con un buen estudio y mandos operacionales de la organización, así que para decidir qué tecnología aplicar primero, habrá que preguntarse qué problema se quiere solucionar, el enfoque correcto es basado en el problema y no en las opciones tecnológicas disponibles.

\section{Referencias}

Arias, M. (2018a,b). Tecnología Blockchain. Trabajo de grado. Bogotá, Colombia: Universidad Militar Nueva Granada. Recuperado de: http://hdl.handle.net/10654/20666

Camargo-Vega, J., Camargo-Ortega, J., \& Joyanes-Aguilar, L. (2015).

Conociendo Big Data. Facultad de Ingeniería, 24(38), 63-77, e-ISSN:

0121-1129. Recuperado de:

https://www.redalyc.org/articulo.oa?id=413940775006

Jacquez-Hernández, M., \& López, V. (2018). Modelos de evaluación de la madurez y preparación hacia la Industria 4.0: una revisión de literatura. Ingeniería Industrial. Actualidad y Nuevas Tendencias, VI(20), 61-78, e-ISSN: 1856-8327. Recuperado de:

https://www.redalyc.org/articulo.oa?id=215057003004

Maquilón, J., Mirete, A., \& Avilés, O. (2017). La Realidad Aumentada (RA).

Recursos y propuestas para la innovación educativa. Revista Electrónica Interuniversitaria de Formación del Profesorado, 20(2), 183203, e-ISSN: 1575-0965. Recuperado de:

https://doi.org/10.6018/reifop/20.2.290971

Rouhiainen, L. (2018). Inteligencia Artificial: 101 cosas que debes saber 
Instituto Internacional de Investigación y Desarrollo Tecnológico Educativo INDTEC, C.A.

DOI: https://doi.org/10.29394/Scientific.issn.2542-2987.2021.6.21.14.267-281

OAI-PMH: http://www.indteca.com/ojs/index.php/Revista Scientific/oai

Ensayo Original / Original Essay

hoy sobre nuestro futuro. ISBN: 978-84-17568-08-5. Madrid, España:

Editorial Planeta, S.A.

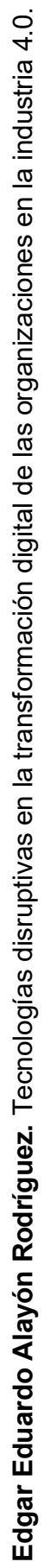

Tascón, M. (2013). Introducción: Big Data. Pasado, presente y

futuro. Telos: Cuadernos de comunicación e innovación, (95), 47-50, eISSN: 0213-084X. Recuperado de:

https://dialnet.unirioja.es/servlet/articulo?codigo $=4423775$ 


\section{Ensayo Original / Original Essay}

\section{Edgar Eduardo Alayón Rodríguez \\ e-mail: ed.alayon@gmail.com}

Nacido en Caracas, Venezuela, el 11 de agosto del año

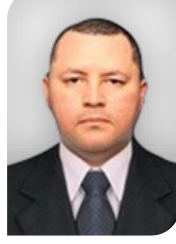
1974. Ingeniero de Sistemas por el Instituto Universitario Politécnico Santiago Mariño (IUPSM); Maestría en Gerencia de Recursos Humanos por la Universidad Nacional Experimental "Rafael María Baralt" (UNERMB); Componente Docente por la Universidad José Gregorio Hernández (UJGH); me he desempeñado como Docente y Coordinador del Programa Nacional de Formación en Informática (PNFI) de la Universidad Nacional Experimental "Rafael María Baralt"; Docente de la Fundación Misión Sucre en el PNFI; así como docente, asesor y jurado evaluador de trabajos de investigación en el Instituto Universitario Politécnico Santiago Mariño (IUPSM).

El contenido de este manuscrito se difunde bajo una Licencia de Creative Commons ReconocimientoNoComercial-Compartirlgual 4.0 Internacional 